\title{
Opaque intraocular lens implantation
}

This article was published in the following Dove Press journal:

Clinical Ophthalmology

7 March 2013

Number of times this article has been viewed

\section{Imran H Yusuf \\ CK Patel}

The Oxford Eye Hospital, West Wing, John Radcliffe Hospital, Headley Way, Headington, Oxford, United Kingdom

Correspondence: CK Patel

The Oxford Eye Hospital, West Wing,

John Radcliffe Hospital, Headley Way,

Headington, Oxford, OX3 9DU,

United Kingdom

Tel +44 I8 65234736

Fax +44 I8 65240097

Email ckpatel@btinternet.com
We read with great interest the recent article by Lee et al, ${ }^{1}$ who described their clinical experience with three patients who underwent primary implantation of Morcher (Stuttgart, Germany) occlusive intraocular lenses (IOLs) across a variety of neuroophthalmic indications. We hope to offer some further insight into these clinical observations in the context of our ongoing research into occlusive IOLs. We feel such a discussion may be of benefit to ophthalmologists managing this group of patients.

Lee et al describe a patient with intractable diplopia who underwent clear lens extraction with implantation of an 80D $6 \mathrm{~mm}$ Morcher occlusive IOL, followed by a second procedure to augment occlusion within the scotopic pupillary margin using two Morcher partial aniridia implants $(50 \mathrm{C})$ and a third procedure to implant a Morcher $80 \mathrm{D}$ occlusive IOL with a $10 \mathrm{~mm}$ optic. ${ }^{1}$ This patient was able to perceive light despite four anatomically continuous occlusive intraocular devices spanning the pupillary aperture. The authors interpret this to suggest the route of postoperative light entry into the eye must be across the intact sclera. Our research indicates a more likely mechanism to explain this perception of light, an understanding of which may be critical to a satisfactory outcome in this group of patients.

We initially reported the serendipitous discovery of successful macular imaging using a combined confocal laser scanning ophthalmoscopy/optical coherence tomography imaging system through the Morcher $85 \mathrm{~F}$ occlusive IOL implanted in a patient with visual confusion. ${ }^{2}$ We analyzed the transmission spectra of all Morcher occlusive IOLs to clarify the mechanism behind this observation, discovering that whilst wavelengths of light below $740 \mathrm{~nm}$ were occluded entirely by the occlusive IOL, there was an exponential increase in light transmission above $750 \mathrm{~nm}$, with approximately $50 \%$ of light transmitted at $780 \mathrm{~nm}$ and $100 \%$ of light transmitted at wavelengths longer than $820 \mathrm{~nm}$ through the Morcher occlusive IOL. ${ }^{3}$ This explained the acquisition of high quality images with combined confocal laser scanning ophthalmoscopy/optical coherence tomography imaging systems which use a near infrared superluminescent diode as their light source.

Near infrared light activates photopigments in long wavelength red cone photoreceptors, resulting in light perception, provided that the stimulus of light is sufficiently bright. Photopic conditions and sources of light with a rich near infrared component are likely to be perceived by patients with near infrared-transmitting occlusive IOLs produced by Morcher. This near infrared window of transmission is the likely primary mechanism underlying refractory light perception in patients implanted with Morcher 
occlusive IOLs, rather than trans-scleral transmission of light through the intact sclerochoroidal tunic, as suggested.

Light perception across a near infrared-transmitting occlusive IOL must be considered a possibility by ophthalmologists in patients undergoing primary implantation with a Morcher occlusive IOL, and patients should be appropriately counseled preoperatively during the process of informed consent. As Lee et al state, patients with neuro-ophthalmic disorders may be very sensitive to any perception of light in the affected eye. Therefore, absolute light occlusion is the primary goal of treatment in this group, and may be critical to a satisfactory clinical outcome for the patient.

The authors report the use of Morcher partial aniridia castellated rings to augment light occlusion around the optic in cases with suspected light leakage due to mesopic or scotopic pupillary dilatation beyond the occlusive optic. Morcher produce occlusive IOLs by adding a tint to monomers prior to polymerization to polymethylmethacrylate. ${ }^{4}$ Morcher partial aniridia castellated rings $(50 \mathrm{C}, 50 \mathrm{E}, 50 \mathrm{~F}$, 96C, 96F, 96E, 96G) are produced from the same material used to manufacture the Morcher range of occlusive IOLs (80D, 81D, 34D, 81F, 85F), so can be considered to transmit near infrared light identically. ${ }^{3}$ Whilst we agree that partial aniridia rings may augment occlusion of light, they cannot create absolute occlusion because of the optical properties of the materials from which they are manufactured, even in the presence of complete and contiguous anatomical occlusion due to apposition of the individual devices.

If a near infrared-transmitting Morcher occlusive IOL (81D, 85F, 34D) with a 6-7 mm occlusive optic is insufficient to produce satisfactory occlusion of light, we suggest that using Morcher 80D IOLs with a $10 \mathrm{~mm}$ optic is preferable to using partial aniridia implants, which may not be stable enough to augment occlusion even with overlapping castellations. Both of these occlusive strategies will not prevent occlusion of near infrared light. Multiple Morcher occlusive intraocular devices, whilst removing nonoccluded regions between prostheses, would not add any occlusive value beyond attenuating the intensity of near infrared light due to optical reflections from their anterior interfaces. A single Morcher occlusive IOL is sufficient to occlude an intense, broad spectrum light source with an output irradiance of $3.73 \times 10^{3} \mathrm{~mW} / \mathrm{cm}^{2}$ within the visible spectrum below $750 \mathrm{~nm}$, with an exponential increase in transmission at longer wavelengths.

A distinction has emerged between the Morcher range of occlusive IOLs which transmit near infrared light, the occlusive polymethylmethacrylate IOL (MS 612, Dr Schmidt
Intraocularlinsen $\mathrm{GmbH}$, Sankt Augustin, Germany), and the occlusive iris-claw IOL (Artisan 201, Ophtec, Boca Raton, FL, USA) which are occlusive to all wavelengths of light. ${ }^{4}$ Therefore, the alternative surgical approach in this setting is to implant an iris-claw occlusive IOL, which has the dual advantage of achieving complete pupillary occlusion, with the use of intracameral Miochol intraoperatively prior to enclavation, making preoperative scotopic pupillometry irrelevant, and simultaneously occluding all wavelengths of light, including in the near infrared range, achieving a more robust state of light occlusion. ${ }^{4}$

We agree that the intraocular risks associated iris fixation such as uveitis, glaucoma, pigment dispersion syndrome, and endothelial cell loss make this implant less attractive as a primary occlusive strategy, but it can offer more definitive occlusion should other black IOLs fail to control symptoms. The MS 612 occlusive IOL should be considered in patients in whom preoperative scotopic pupillometry is appropriate for a $6 \mathrm{~mm}$ occlusive optic and robust occlusion of light is essential. This implant will not transmit near infrared light, even under intense photopic conditions. ${ }^{4}$

The third reported case in the series describes a patient with compressive optic neuropathy occurring secondary to a displaced intracranial vascular clip for a posterior communicating artery aneurysm. Intriguingly, this patient did not perceive light after implantation of a near infrared-transmitting IOL. This may be due to preferential loss of red perception typical of optic neuropathies, with impairment of the ability to detect near infrared light using long wavelength red cone photoreceptors and their connecting retinal ganglion cells. In the setting of an optic neuropathy, occlusion of visible spectrum light by the Morcher occlusive IOL may have been sufficient to occlude all light perception. A near infraredtransmitting occlusive IOL may be the prosthesis of choice for primary occlusion in these patients, provided red color perception is impaired on the Farnsworth-Munsell 100 hue test preoperatively. This will permit combined confocal laser scanning ophthalmoscopy/optical coherence tomography imaging for posterior segment examination without risking refractory light perception.

We hope this discussion aids the ophthalmologist caring for patients eligible for occlusive IOL implantation, adding to the clinical lessons reported by Lee et al. ${ }^{1}$ Patient factors must be considered when selecting the appropriate primary occlusive intraocular implant to use in each case, and when considering the optimum surgical strategy for secondary procedures should the patient experience refractory light perception through the primary occlusive implant. 
Understanding the mechanisms of light transmission across occlusive IOLs is critical to ensuring a satisfactory clinical outcome in each eligible patient.

\section{Disclosure}

None of the authors have any financial interest in any product, method, or material mentioned in this correspondence.

\section{References}

1. Lee RM, Dubois VD, Mavrikakis I, et al. Opaque intraocular lens implantation: a case series and lessons learnt. Clin Ophthalmol. 2012;6:545-549.

2. Patel CK, Yusuf IH, Menezo V. Imaging the macula through a black occlusive intraocular lens. Arch Ophthalmol. 2010;128:1374-1376.
3. Yusuf IH, Peirson SN, Patel CK. Occlusive IOLs for intractable diplopia demonstrate a novel near-infrared window of transmission for SLO/ OCT imaging and clinical assessment. Invest Ophthalmol Vis Sci. 2011;52:3737-3743.

4. Yusuf IH, Peirson SN, Patel CK. Inability to perform posterior segment monitoring by scanning laser ophthalmoscopy or optical coherence tomography with some occlusive intraocular lenses in clinical use. $J$ Cataract Refract Surg. 2012;38:513-518. 


\section{Authors' Reply}

\section{Richard MH Lee' \\ Vincent DJ-P Dubois' \\ loannis Mavrikakis' \\ Salim Okera' \\ Gerard Ainsworth' \\ Sarah Vickers' \\ Christopher SC Liu',}

'Department of Ophthalmology, Sussex Eye Hospital, Brighton, ${ }^{2}$ Tongdean Eye Clinic, Hove, UK

Correspondence: Christopher SC Liu

Department of Ophthalmology, Sussex Eye Hospital, Eastern Road, Brighton, BN2 5BF, UK

$\mathrm{Tel}+44$ I $273606 \mid 26$

Fax+441273693674

Email cscliu@aol.com

We thank Yusuf and Patel for their interest in our article and their insightful comments. They have previously published their findings on transmission of near infrared light through an occlusive intraocular lens (IOL). ${ }^{1}$ This is useful because it is not possible to examine the posterior pole on slit-lamp biomicroscopy and because combined confocal laser scanning ophthalmoscopy/optical coherence tomography imaging systems use a near infrared superluminescent diode as their light source, allowing for imaging of the posterior pole through an occlusive IOL. Although near infrared transmission may be a mechanism for light perception through an occlusive IOL, this has not been supported by clinical evidence in patients who have previously undergone phacoemulsification surgery with occlusive IOL insertion. We are planning to examine this in several of our patients in a future study.

We reported the use of Morcher partial aniridia castellated rings to augment light occlusion around the optic in a patient with suspected light leakage due to mesopic or scotopic pupillary dilatation beyond the occlusive optic. ${ }^{2} \mathrm{We}$ were not advocating and do not advocate planned insertion of multiple IOL devices or performing multiple procedures. The aim of our paper was to share our learning curve of using occlusive IOL and to offer our advice for the benefit of other ophthalmologists considering occlusive IOL implantation as a treatment strategy for their patients. Having learned from this patient the necessity of scotopic pupillometry, we were able to order a custom-made device for our third patient and thereby avoid the problems we had faced with our earlier patient. While a Morcher $80 \mathrm{D}$ IOL with a $10 \mathrm{~mm}$ optic could be recommended as a standard secondary IOL if the patient perceives light around the IOL in scotopic conditions, implantation of such an IOL would require a larger wound to be made until such time that foldable occlusive IOLs are developed. We believe that including scotopic pupillometry preoperatively allows avoidance of light leakage for those with large pupils by way of ordering a sufficiently large, custom-made occlusive implant, if necessary.

Posterior vaulting of IOLs with forward angulated haptics were designed to put pressure on the posterior capsule to reduce the incidence of posterior capsule opacification. This is less important with occlusive devices. In fact, with reduced or zero angulation, there would be less anteroposterior gap between the iris and "optic" planes, reducing the possibility of light leakage. We would caution against inverting an occlusive device back to front, because that will risk pupil block and angle closure. We currently prefer "black-on-clear" IOL insertion, with both an occlusive IOL and a clear IOL placed within the bag. ${ }^{3}$ This allows safe removal of the black occlusive device should the need arise, averting the need for IOL exchange, and we have shown that dual polymethylmethacrylate IOLs in the bag have good long-term stability and centration, with minimal interlenticular opacification. ${ }^{4}$ Scotopic pupillometry is also essential prior to planning a "black-on-clear" procedure. Although we agree that an anterior chamber or iris fixation device could be considered in a phakic eye or if symptoms are not controlled with an occlusive IOL, we do not recommend these due to the associated complications already discussed in our article. ${ }^{2}$

In conclusion, we agree that strict preoperative counseling is important in these patients regarding the fact that we are operating on a healthy eye, and postoperative examination of the posterior pole will not be possible other than by imaging techniques. Patients should also be made aware that in some cases they may require additional procedures and still perceive light, although the mechanism by which this occurs is debatable. There is currently no clear evidence to recommend one IOL device, manufacturer, or material over another for any individual patient. However, from our experience, we now prefer dual polymethylmethacrylate IOLs to be both placed within the bag, with an occlusive IOL placed in front of a clear IOL, having first done preoperative scotopic pupillometry.

\section{Disclosure}

The authors report no conflicts of interest in this work.

\section{References}

1. Patel CK, Yusuf IH, Menezo V. Imaging the macula through a black occlusive intraocular lens. Arch Ophthalmol. 2010;128: 1374-1376. 
2. Lee RM, Dubois VD, Mavrikakis I, et al. Opaque intraocular lens implantation: a case series and lessons learnt. Clin Ophthalmol. 2012;6:545-549.

3. Byard SD, Lee RM, Lam FC, Simpson AR, Liu CS. Black-on-clear piggyback technique for a black occlusive intraocular device in intractable diplopia. J Cataract Refract Surg. 2012;38:5-7.
4. Gomaa A, Lee RM, Liu CS. Polypseudophakia for cataract surgery: 10-year follow-up on safety and stability of two poly-methyl-methacrylate (PMMA) intraocular lenses within the capsular bag. Eye (Lond). 2011;25: 1090-1093.

\section{Publish your work in this journal}

Clinical Ophthalmology is an international, peer-reviewed journal covering all subspecialties within ophthalmology. Key topics include: Optometry; Visual science; Pharmacology and drug therapy in eye diseases; Basic Sciences; Primary and Secondary eye care; Patien Safety and Quality of Care Improvements. This journal is indexed on

Submit your manuscript here: http://www.dovepress.com/clinical-ophthalmology-journal

\section{Dovepress}

PubMed Central and CAS, and is the official journal of The Society of Clinical Ophthalmology (SCO). The manuscript management system is completely online and includes a very quick and fair peer-review system, which is all easy to use. Visit http://www.dovepress.com/ testimonials.php to read real quotes from published authors. 